\title{
Characteristics on micro-biofabrication by patterning with electrostatically injected droplet
}

\author{
Shinjiro Umezua,b, , Hitoshi Ohmori (1) ${ }^{\text {a }}$ \\ ${ }^{a}$ RIKEN, Saitama, Japan \\ ${ }^{b}$ Tokai University, Kanagawa, Japan
}

Micro biofabrication technologies have been developing aiming to fabricate 3D artificial organs, 3D scaffolds, and complex tissue structures. We are now developing a new inkjet bio-printing method via electrostatic phenomenon. The merits of the new method are of high resolution, and of ability to eject highly viscous liquid and media. In this paper, we attempted to apply the proposed method for precision printing cells and biomaterials. Living cells and scaffolds have successfully been printed and the biochemical characteristics have been investigated. A 3D cell structure which had a cavity to create blood vessels has also successfully fabricated by this method.

Keywords: Rapid prototyping; Biomedical; Micro structure

\section{Introduction}

Biomanufacturing technologies are emergently pursued to improve QOL(Quality Of Life) by replacing original parts in human body with artificial ones fabricated by means of engineering methodologies. There are several examples developing artificial joints and implants using bio-compatible materials with specific surface processing methods [1] [2] [3] [4]. On the other hand, in order to fabricate three-dimensional (3D) artificial organs containing live cells with fine internal structures, new micro biofabrication methods must be developed. MEMS technology [5], in general, is suitable to fabricate complex 3D micro/nano structures. This technology needs large scale fabrication facilities and also photo masks to generate micro/nano scaffold structures by lithography. Moreover, a vacuum condition is not preferable for keeping cells alive during putting them into the micro scaffold structures. Among another technology, 3D Collagen Sponge Method [6] is suggested to fabricate $3 \mathrm{D}$ cell structures. When a $3 \mathrm{D}$ collagen sponge is put into a medium with cells, cells are attached to the surface of the sponge. This technology is applied for investigation of growth of neuron cells. However, it is difficult to control the amount in attachment and position of the cells. Cell Sheet Technology [7] is now being applied to fabricate 3D cell structures instead. When several kinds of cell sheets are laminated, a simple 3D cell structure is fabricated. Blood vessels play an important role in our body because nutrition is transported through blood vessels. In case that cells are $200 \mu \mathrm{m}$ far from blood vessels, they will die because of nutrition shortage. Thus, the position of cells must be controlled precisely to fabricate complex 3D cell structures.

Under those backgrounds, printing technology is recently highly focused to solve those mentioned subjects. Direct Printing Method [8] is suitable to print cells and biomaterials (scaffolds) in terms of resolution and positioning precision, even though viscosity of biomaterials is relatively high. Furthermore Drop-OnDemand (DOD) printing [9] [10] is possible to fabricate precision bio structures. Thus, an inkjet printing technology can be used for 3D fabrication to pile 2D printed layers. Skull and born have successfully been fabricated by using inkjet technology [11].
Piezo and thermal printing types belong to DOD printing. It has been found that ejected cells had been alive in spite of high pressure or heat during printing. Commercial piezo inkjet printing technology was tried to be applied for 3D positioning of calcium alginate which contained living cells [12], though, it could eject only scaffolds with low viscosity [13].

Precision artificial organs can be fabricated when high resolution to print in control of cell position and ability to eject highly viscous liquid can be achieved due to high viscosity of most biomaterials to be used. We are now developing an innovative inkjet bio-printing method via electrostatic phenomenon to solve the problem. Patterning with ELectrostatically Injected Droplet (PELID) method as a new bio printing has been proposed to give merits on 3D bio fabrication; those are of high resolution in printing cells and biomaterials and of ability to eject highly viscous liquid [14] [15].

In this study, we investigated the characteristics in droplet formation mode of this new printing method. Applying this method, 3D cell structures which consist of living cells and scaffolds have successfully been fabricated. It has also been confirmed that the cells could be alive and even grow after the fabrication of the 3D cell structures by this method.

\section{Methodology and experimental procedure}

\subsection{Fundamental characteristics}

An experimental set-up illustrated in Fig. 1 was constructed to investigate drop ejection modes in patterning phenomena with electrostatically injected droplet. The capillary tube made of silica coated by polyimide was equipped with a bottom of a syringe. Ion-conductive liquid is poured into the syringe. This tube with the liquid is hanged down perpendicular to a plate electrode made of stainless steel. Voltage is applied by a function generator and a high voltage amplifier; the capillary tube was charged positively, the plate electrode was charged negatively. The formation of droplet was observed by a high speed camera with lighting to observe transient formation and ejection of droplets. The gap between the capillary tube and the plate electrode was 
adjusted by a Z-stage and the plate electrode was moved in X and $\mathrm{Y}$ directions with two linear motors.

\subsection{Application for biofabrication}

The experiment was conducted to observe the suitability of the new method in printing cells precisely and ejecting highly viscous scaffolds. Fabrication steps are shown in Fig. 2. A 3D structure can be fabricated by the patterning cells and scaffolds together with a cultivation process.

The experimental set-up to pattern 3D cell structure is modified from Fig. 1. As shown in figure 3 and 4, dish which is filled with medium is set on the plate electrode. When syringe is filled with cells and/or collagen, 2D printing is carried out. In case that syringe is filled with aqueous solution of sodium alginate and dish is slightly filled with aqueous solution of calcium chloride, calcium alginate gel is generated in dish when the droplets of aqueous solution of sodium from the syringe are ejected to the dish filled with the calcium chloride.

When Ca ion was put into the aqueous solution of sodium alginate, gelification was took place because of cross-linking. The viscosity of the aqueous solution of sodium alginate (10\% wt) was $30 \mathrm{mPa} \cdot \mathrm{s}$ at 20 degrees $\mathrm{C}$.

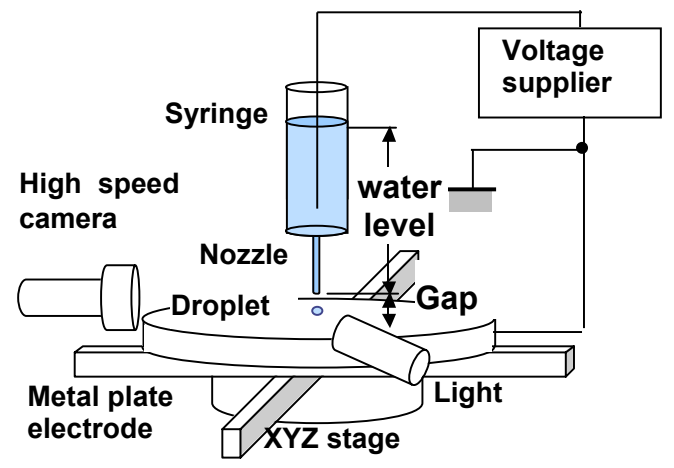

Fig. 1. Experimental set-up.

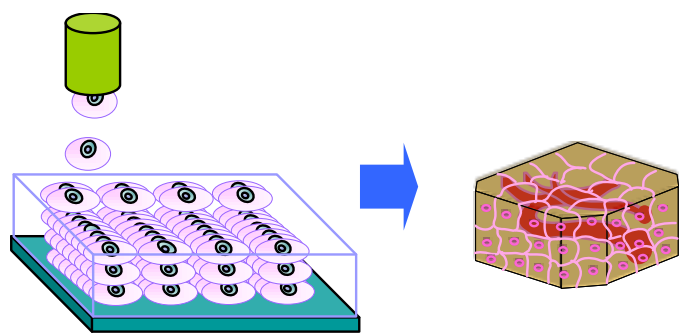

(a) Pattern cells and scaffolds (b) Cultivate the patterned structure Fig. 2. Fabrication process of 3D cell structure.
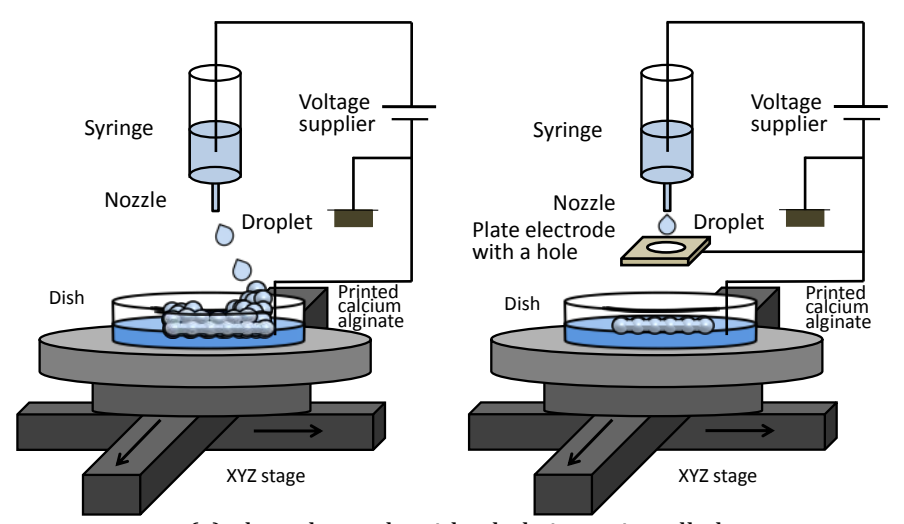

(a) plate electrode with a hole is not installed

(b) plate electrode with a hole is installed

Fig. 3. Experimental set-up to pattern 3D cell structure and printed structures.

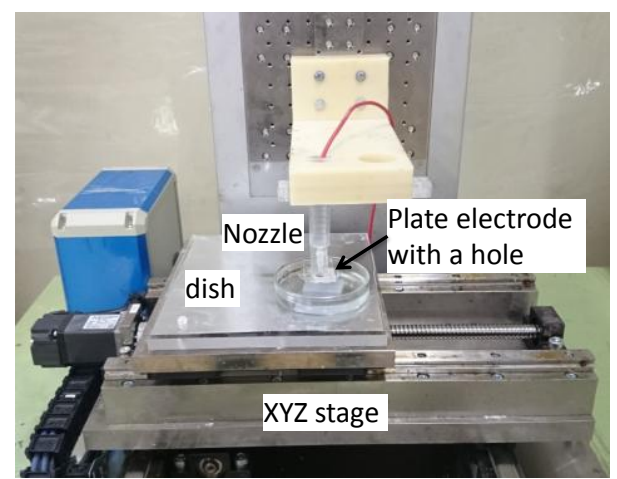

Fig. 4. Photograph of the experimental set-up in case that the plate electrode with a hole is installed

Even when the printed 3D cell structure becomes thick, the air gap is controlled to be constant by Z-stage. When the air gap between nozzle and the printed structure is short, the Taylor cone is inclined to the edge due to the electric concentration. From this reason, it is difficult to fabricate complex 3D structure precisely in a normal way. As to solve this problem, a plate electrode with a hole is devised between the nozzle and the dish in order to control the droplet direction more precisely. Precision print has successfully achieved though the air gap is short because the electric field around the tip of nozzle is controlled to be constant with the hole of the plate.

\section{Experimental results and discussions}

\subsection{Fundamental characteristics}

Firstly we classify the droplet formation into following three modes shown in Fig. 5. These three modes are determined by the applied voltage and the air gap.

Drop mode: At the dark discharge region, $3-4 \mathrm{kV}$, a drop is formed at the tip of the tube. This becomes large gradually and drops finally. The diameter of the drop is several times larger than that of the tube diameter.

Droplet mode: At the beginning of the corona discharge, $4-4.5 \mathrm{kV}$, a Taylor cone [16] is formed at the end of the tube and the tip of the cone periodically separates from the cone to form a very small droplet. The size is from several $\mu \mathrm{m}$ to several tens $\mu \mathrm{m}$ in diameter. Droplet which size was smaller than the nozzle size was formed because of balance between relatively strong electrostatic force, gravity force, and surface tension. Precision printing could be achieved because the droplet size of commercial inkjet printer was the same as the nozzle size. When the highly viscous liquid was used, pipe friction was decreased by enlargement of nozzle and small droplet was formed at the tip of the Taylor cone. Fig. 6 shows the print samples utilizing the droplet mode. This figure

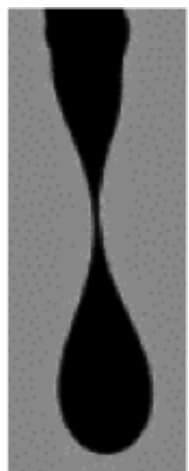

(a) Drop mode (b) Droplet mode

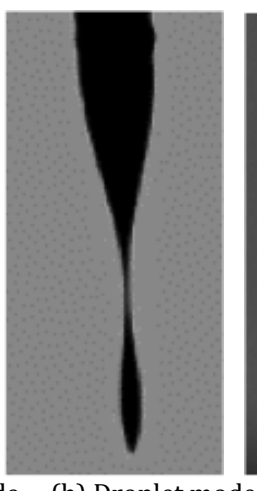

Fig. 5. Mode of droplet formation. (nozzle size is $200 \mu \mathrm{m}$.)

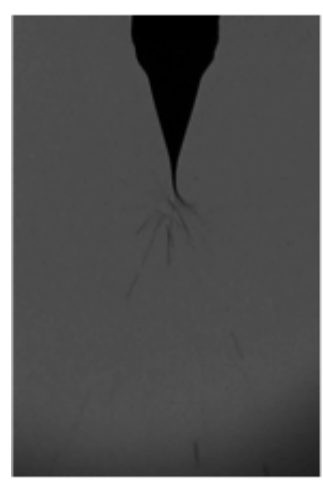

(c) Spray mode 


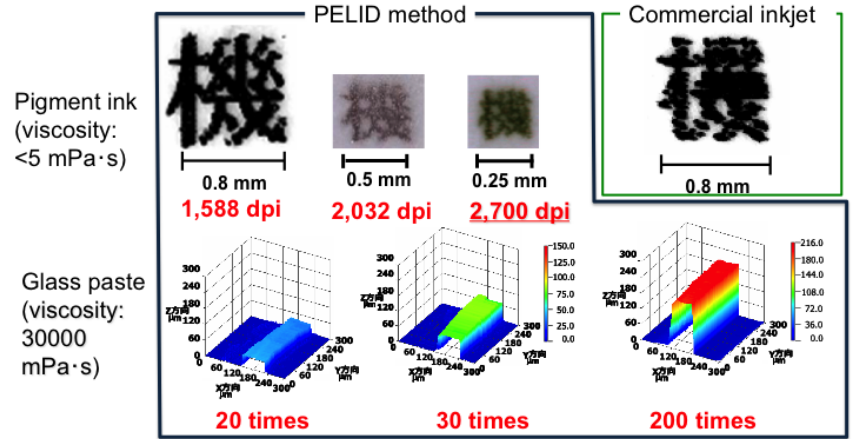

Fig. 6. Print sample. (upper: print samples of Chinese character "mechanical" with pigment ink, lower: print samples of glass paste)

indicates two merits; higher resolution than a commercial inkjet printer's, and ability to eject highly viscous liquid.

Spray mode: with the higher voltage, droplets disperse at a wide angle like mist from the Taylor cone. The size of droplet is from sub $\mu \mathrm{m}$ to several $\mu \mathrm{m}$ in diameter.

These results indicate that droplet mode is suitable to apply for high resolution patterning, and spray mode is suitable to apply for precision coating.

\subsection{Cell print}

We investigated an influence in living cells by application of high voltage. Fig. 7 shows photographs of printed cells when one day has passed after ejection of Madin-Darby Canine Kidney (MDCK) cells utilizing the proposed printing method. Fig. 8 shows growth curve of the MDCK cells. Before day 4, number of the cells was increased. At day 4, cells were spread all over the dish, and then confluence-induced apoptosis took place. So, the number of cells was drastically decreased. These results indicate that the most cells were alive in spite of high voltage application because electric current does not flow inside the cells but the cells are only charged. Cells were living in case that the diameter of the tube was over $100 \mu \mathrm{m}$. In case that the diameter of the tube was less than $50 \mu \mathrm{m}$, it was difficult to eject continuously because cell attachment takes place inside the tube. Fig. 9 and 10 show

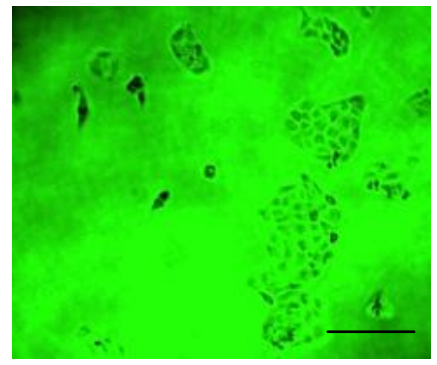

(a) 1day has passed

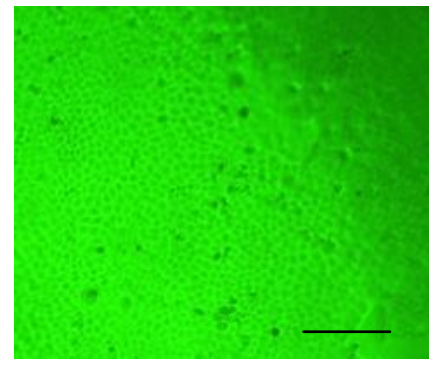

(b) 3days has passed
Fig. 7. Cell growth of printed MDCK cells.

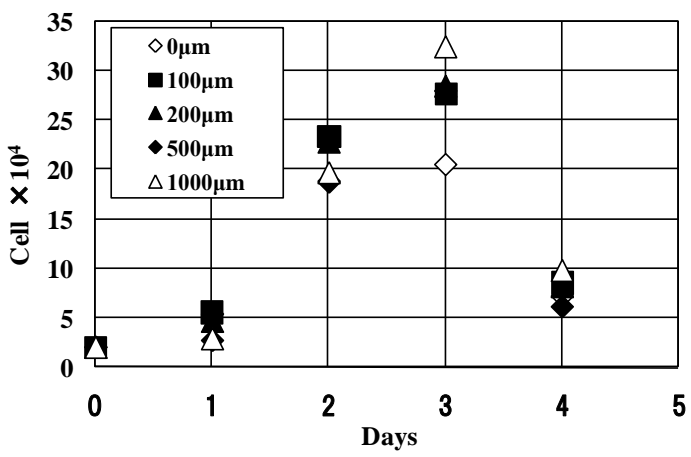

Fig. 8. Growth curve of MDCK cells on collagen layer.
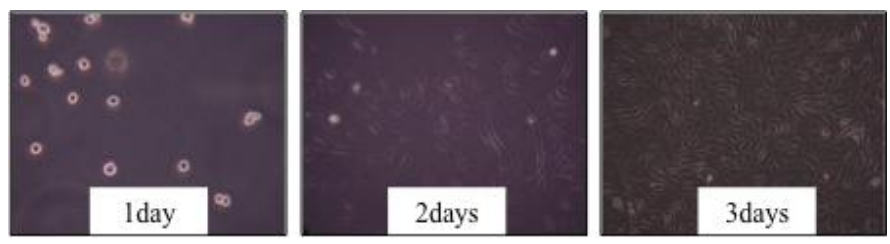

Fig. 9. Cell growth of printed HH cells.
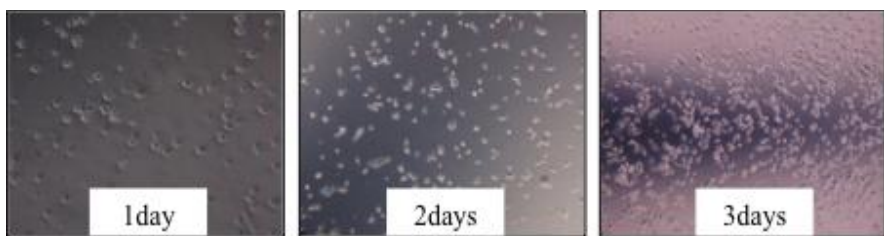

Fig. 10. Cell growth of printed 293T cells.

growths of printed $\mathrm{HH}$ cells and 293T cells. These results indicate that it is possible to print cells utilizing PELID method in spite of high voltage application.

\subsection{Scaffolds printing}

Scaffolds between cells are necessary to fabricate 3D cell structures by bonding cells strongly enough. Collagen, gelatin, and calcium alginate gel is well known scaffolds. Collagen is the main structural protein of the various tissues in animals. Gelatin is an irreversibly hydrolysed form of collagen. Calcium alginate gel is usually used as micro bio capsule and the stiffness is high.

Fig. 11 shows line width of printed gelatin. In case of short air gap, printed gelatin line is narrow because Taylor cone becomes sharpened.. In the area with the voltage of less than $1.7 \mathrm{kV}$, the higher the voltage was, the narrower the line width became. On the other hand, in the area with the voltage of $1.7 \mathrm{kV}$ and over, the higher the voltage was, the wider the line width became. Taylor cone becomes round because stable corona discharge takes place. The line width was several tens $\mu \mathrm{m}$. Line of gelatin should be narrower because gelatin is aimed to be used as scaffolds. Fig. 11 indicates that when the air gap is small, the line is narrow. Fig. 12 shows photograph of printed gelatin in case that the air gap is 0.1 $\mathrm{mm}$ and the applied voltage is $1.7 \mathrm{kV}$. The line width is more or less $10 \mu \mathrm{m}$. The tendency of printing collagen is the same as that of printing gelatin.

\subsection{Print of $3 D$ cell structure with blood vessel cave}

Calcium alginate gel is generally used as scaffolds because the stiffness of calcium alginate gel is much higher than that of collagen. Hence, it is easy to fabricate complex 3D structures with calcium alginate gel. 3D structures which consisted of only cells and calcium alginate has already been reported [14]. However, complex 3D structures need caves inside to create blood vessels

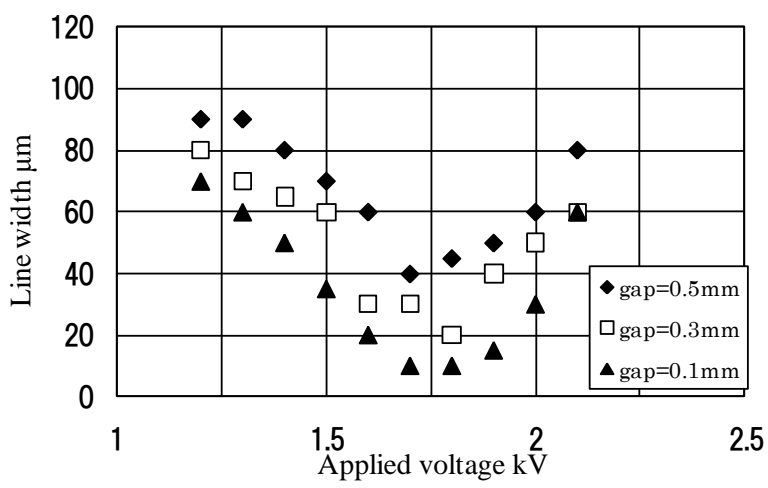

Fig. 11. Line width of printed gelatin when applied voltage and air gap are changed. 


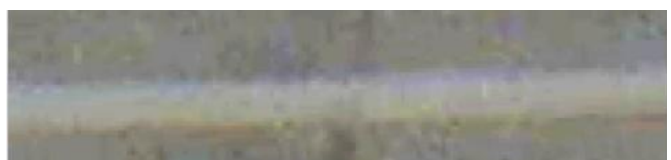

Fig. 12. Printed gelatin line.

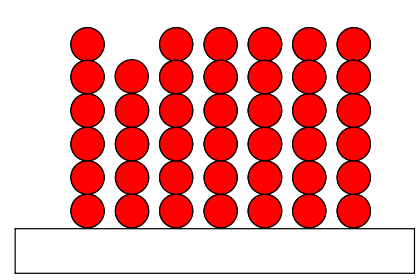

- calcium Alginate

Fig. 13. 3D structure containing only calcium alginate.

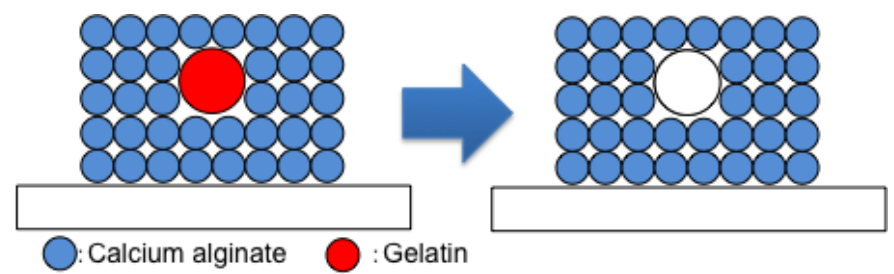

(a) Fabrication process

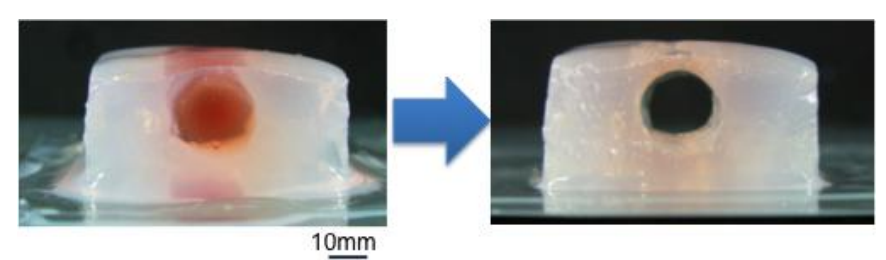

(b) 3D structure that has I-shape cave

Fig. 14. Process to fabricate complex 3D structure having cave for blood vessel.

like real 3D cell structures. When calcium alginate is printed on free space, a cave is not formed because ejected calcium alginate layer cannot remain on free space but can make only a dimple shown in Fig. 13. Fig. 14 (a) shows the new process to fabricate a complex 3D structure which has a cave. We use gelatine as a sacrificial layer. Upper layer on gelatine is patterned with calcium alginate while gelatin works as a support. When the patterned 3D structure is put into hot water, gelatine is removed and a cave is formed inside the 3D structure. Fig. 14 (b) show fabricated 3D structure that has I-shape cave. The shape and diameter of the cave were controlled by the CAD data. These results suggest that complex 3D cell structures can be fabricated by the proposed printing method.

\section{Conclusions}

It has been proved that small droplets can be ejected by electrostatic injecting phenomenon. The merits are of high resolution and of ability to eject highly viscous liquid. This phenomenon could be successfully applied to print living cells and several kinds of scaffolds. In spite of high static voltage application, it was found that the cell growth has not been damaged. The scaffolds of which are collagen, gelatin, and alginate gel could be printed. The width of printed gelatin line was more or less $10 \mu \mathrm{m}$. It was narrow enough for cell printing because the diameter of cell is about 30 to $50 \mu \mathrm{m}$. Alginate gel is good biomaterial to fabricate 3D structures because of high stiffness. We tried to fabricate a 3D cell structure which has cave as blood vessel inside. Blood vessels play an important role in our body because nutrition is transported through them. When cells are $200 \mu \mathrm{m}$ far from blood vessels, cells die because of nutrition shortage problem. So, 3D cell structure which has blood vessels must be fabricated. However, it is difficult to fabricate a 3D cell structure which has a cave. When calcium alginate is printed on free space, cave cannot be formed because ejected calcium alginate cannot remain on free space but make only a dimple. So, Gelatin was used to solve this problem. We used gelatine as a sacrificial layer. Upper layer on gelatine was successfully printed precisely because gelatine has worked as a support. When the printed 3D structure is put into hot water, gelatine was removed and a cave was formed in the 3D structure. Design of cave depends on CAD data of Gelatin layer. Applying this technique, it was suggested that complex 3D cell structures which have blood vessels can be fabricated.

\section{Acknowledgements}

This work is supported by JST A-STEP and MEXT/JSPS Grantsin-Aid for Scientific Research C.

\section{References}

[1] Mitsuishi, M., Cao, J., Bártolo, P., Friedrich, D., Shih, A. J., Rajurkar, K., Sugita, N. Harada, K., 2013, Biomanufacturing, Annals of the CIRP, 62/2:585-606.

[2] Bartolo, P., Kruth, J-P., Silva, J., Levy, G., Malshe, A., Rajurkar, K., Mitsuishi, M., Ciurana, J., Leu M., 2012, Biomedical Production of Implants by Additive Electro- Chemical and Physical Processes, Annals of the CIRP, 61/2:635-655.

[3] Ohmori, H., Mizutani, M., Kaneeda, T., Abe, N., Okada, Y., Moriyama, S., Hisamori, N., Nishimura, N., Tsunashima, Y., Tanaka, J., Kuramoto, K., Ezura, A., 2013, Surface generating process of artificial hip joints with hyper-hemispherical shape having higher smoothness and biocompatibility, Annals of the CIRP, 62/1:579-582.

[4] Ohmori, H., Katahira, K., Nagata, J., Mizutani, M., Komotori, J., 2002, Improvement of Corrosion Resistance in Metallic Biomaterials by a New Electrical Grinding Technique, Annals of the CIRP, 51/1:491-494.

[5] Hayashi, T., Shibata, T., Kawashima, T., Makino, E., Mineta, T., Masuzawa, T., 2008, Photolithography system with liquid crystal display as active gray-tone mask for 3D structuring of photoresist, Sensors and Actuators A: Physical, 144/2:381-388.

[6] Chen, C. H., Kuo, S. M., Liu, G. S., Chen, W. U., Chuang, C. W., Liu, L. F., 2012, Enhancement of neurite outgrowth in neuron cancer stem cells by growth on 3 D collagen scaffolds, Biochemical and Biophysical Research Communications, 428/1:68-73.

[7] Takahashi, H., Shimizu, T., Nakayama, M., Yamato, M., Okano, T., 2013, The use of anisotropic cell sheets to control orientation during the self-organization of 3D muscle tissue, Biomaterials, 34:7372-7380.

[8] Bartolo, P., Domingos, M., Gloria, A., Ciurana, J., 2011, BioCell Printing: Integrated automated assembly system for tissue engineering constructs, Annals of the CIRP, 60/1:271-274

[9] Liu, Z., Su, Y., Varahramyan, K., 2005, Inkjet-printed silver conductors using silver nitrate ink and their electrical contacts with conducting polymers, Thin Solid Films, 478/1:275-279.

[10] Utela, B., Stoti, D., Anderson, R.. Ganter, M., 2008, A review of process development steps for new material systems in three dimensional printing (3DP), Journal of Manufacturing Processes, 10/2:96-104.

[11] Bose, S., Vahabzadeh, S., Bandyopadhyay, A., 2013, Bone tissue engineering using 3D printing, Materialstoday, 16/12:496-504.

[12] Henmi, C., Nakamura, M., Nishiyama, Y., Yamaguchi, K., Mochizuki, S., Takiura, K., Nakagawa, H., 2007 Development of an effective three dimensional fabrication technique using inkjet technology for tissue model samples, Alternatives to Animal Testing and Experimentation, 14:689-692.

[13] Saunders, R. E., Gough, J. E., Derby, B., 2008, Delivery of human fibroblast cells by piezoelectric drop-on-demand inkjet printing, Biomaterials, 29/2:193-203.

[14] Umezu, S., Kitajima, T., Ohmori, H., Ito, Y., 2011, Fundamental characteristics of printed cell structures utilizing electrostatic inkjet phenomena, Sensors and Actuators A: Physical, 166/2:251-255.

[15] Umezu, S., Hatta, T., Ohmori, H., 2013, Fundamental characteristics of bioprint on calcium alginate gel, Japanese Journal of Applied Physics, 52/2: 05DB20.

[16] Taylor, G., 1964, Disintegration of water drops in an electric field. Proceedings of the Royal Society of London Series A: Mathematical and physical sciences, 280:383-397. 